\title{
Erratum to: An empirical central limit theorem for intermittent maps
}

\section{J. Dedecker}

Published online: 25 October 2011

(C) Springer-Verlag 2011

\section{Erratum to: Probab. Theory Relat. Fields (2010) 148:177-195 DOI 10.1007/s00440-009-0227-5}

There are two problems in this paper.

First, the definition of the coefficient $\beta_{2}(n)$ is in fact too restrictive. Secondly, there is a wrong argument in the proof of the main result, Theorem 2.1.

In this erratum, we give the correct definition of the coefficient $\beta_{2}(n)$, as it was introduced in Dedecker and Prieur [1], and we explain how to fix the proof of Theorem 2.1.

The first paragraph is devoted to the definition of the coefficients. In the second paragraph, we give a slightly more general Rosenthal-type inequality than that given in Proposition 3.1, which will be used to fix the proof of Theorem 2.1. In the third paragraph, we explain the changes in the proof of Theorem 2.1.

\section{Definition of the coefficients}

Keeping the same notations as in Definition 2.1 page 180 , the term $b_{2}\left(\mathcal{M}_{l}, k\right)$ should be

$$
b_{2}\left(\mathcal{M}_{l}, i, j\right)=\sup _{(s, t) \in \mathbb{R}^{2}}\left|P_{\left(X_{i}, X_{j}\right) \mid \mathcal{M}_{l}}\left(f_{t}^{(0)} \otimes f_{s}^{(0)}\right)-P_{\left(X_{i}, X_{j}\right)}\left(f_{t}^{(0)} \otimes f_{s}^{(0)}\right)\right| .
$$

The online version of the original article can be found under doi:10.1007/s00440-009-0227-5.

J. Dedecker $(\varangle)$

Laboratoire MAP5, UMR CNRS 8145, Université Paris descartes, Sorbonne Paris Cité,

45 rue des saints pères, 75270 Paris cedex 06, France

e-mail: jerome.dedecker@parisdescartes.fr 
and the correct definition of $\beta_{2}(k)$ should be

$$
\beta_{2}(k)=\max \left\{\beta_{1}(k), \sup _{i>j \geq k} \mathbb{E}\left(\left(b_{2}\left(\mathcal{M}_{0}, i, j\right)\right)\right)\right\},
$$

which is exactly the definition given by Dedecker and Prieur [1].

\section{The Rosenthal inequality}

The inequality given in Proposition 3.1 is correct, but we shall use a slightly more general version. We use the convention $\sum_{i=j}^{k} a_{i}=0$ if $j>k$, and we use the notation $(k)_{+}=k \mathbf{1}_{k>0}$.

Here is the new version of Proposition 3.1 (note that the previous version can be obtained by taking $d_{1}=d_{2}=\cdots=d_{n}=0$ in this new version).

Proposition 3.1 Let $X_{1}, \ldots, X_{n}$ be $n$ real-valued random variables in $\mathbb{L}^{p}$ for some $p \in[2,3]$, with zero expectation, and let $d_{1}, \ldots, d_{n}$ be $n$ real numbers. Let $S_{n}=$ $X_{1}+\cdots+X_{n}$. For $1 \leq i \leq n$, let $\mathcal{F}_{i}=\sigma\left(X_{1}, \ldots, X_{i}\right)$. For any $1 \leq N \leq n$, the following inequality holds

$$
\left\|S_{n}\right\|_{p} \leq\left(2(p-1) \sum_{i=1}^{n} \gamma_{i}\right)^{1 / 2}+\left(\sum_{i=1}^{n} \mathbb{E}\left(\left|X_{i}\right|^{p}\right)+p(p-1) \sum_{i=1}^{n}\left(\delta_{i, 1}+\delta_{i, 2}+\delta_{i, 3}\right)\right)^{1 / p}
$$

where

$$
\begin{aligned}
\gamma_{i} & =\frac{1}{2} \mathbb{E}\left(X_{i}^{2}\right)+\sum_{j=(i-N)_{+}+1}^{i-1}\left|\mathbb{E}\left(X_{i} X_{j}\right)\right|+\sum_{j=1}^{i-N}\left\|X_{j} \mathbb{E}\left(X_{i} \mid \mathcal{F}_{j}\right)\right\|_{p / 2}, \\
\delta_{i, 1} & =\sum_{j=(i-N)_{+}+1}^{i-1} \sum_{l=(2 j-i)_{+}+1}^{j}\left\|\left|X_{l}\right|^{p-2}\left|X_{j}\right| \mathbb{E}\left(X_{i} \mid \mathcal{F}_{j}\right)\right\|_{1}, \\
\delta_{i, 2} & =\sum_{j=(i-N)_{+}+1}^{i-1} \sum_{l=1}^{(2 j-i)_{+}}\left\|\left|X_{l}-d_{l}\right|^{p-2} \mathbb{E}\left(X_{i} X_{j}-\mathbb{E}\left(X_{i} X_{j}\right) \mid \mathcal{F}_{l}\right)\right\|_{1}, \\
\delta_{i, 3} & =\frac{1}{2} \sum_{j=1}^{i-1}\left\|\left|X_{j}-d_{j}\right|^{p-2} \mathbb{E}\left(X_{i}^{2}-\mathbb{E}\left(X_{i}^{2}\right) \mid \mathcal{F}_{j}\right)\right\|_{1} .
\end{aligned}
$$

Now, the Remark 3.1 following Proposition 3.1 should be written as follows (note that the indices in the definition of the term $\delta_{2}$ of the previous version of Remark 3.1 were wrong, and have been replaced by the correct indices).

Remark 3.1 Assume that the $X_{i}$ 's of Proposition 3.1 are taken from a stationary sequence $\left(X_{i}\right)_{i \in \mathbb{Z}}$, and let $\mathcal{M}_{i}=\sigma\left(X_{k}, k \leq i\right)$. Let also $d_{1}=d_{2}=\cdots=d_{n}=d$ in Proposition 3.1. One has $\gamma_{i} \leq \tilde{\gamma}, \delta_{i, 1} \leq \delta_{1}, \delta_{i, 2} \leq \delta_{2}$ and $\delta_{i, 3} \leq \delta_{3}$, with 


$$
\begin{aligned}
\tilde{\gamma} & =\frac{1}{2} \mathbb{E}\left(X_{0}^{2}\right)+\sum_{k=1}^{N-1}\left|\mathbb{E}\left(X_{0} X_{k}\right)\right|+\sum_{k=N}^{n-1}\left\|X_{0} \mathbb{E}\left(X_{k} \mid \mathcal{M}_{0}\right)\right\|_{p / 2}, \\
\delta_{1} & =\sum_{l=1}^{N-1} \sum_{k=0}^{l}\left\|\left|X_{0}\right|^{p-2}\left|X_{k}\right| \mathbb{E}\left(X_{k+l} \mid \mathcal{M}_{k}\right)\right\|_{1}, \\
\delta_{2} & =\sum_{l=1}^{N-1} \sum_{k=l}^{n}\left\|\left|X_{0}-d\right|^{p-2} \mathbb{E}\left(X_{k} X_{k+l}-\mathbb{E}\left(X_{k} X_{k+l}\right) \mid \mathcal{M}_{0}\right)\right\|_{1}, \\
\delta_{3} & =\frac{1}{2} \sum_{k=1}^{n-1}\left\|\left|X_{0}-d\right|^{p-2} \mathbb{E}\left(X_{k}^{2}-\mathbb{E}\left(X_{k}^{2}\right) \mid \mathcal{M}_{0}\right)\right\|_{1} .
\end{aligned}
$$

The proof of this new Proposition 3.1 is almost identical to the proof of the previous version. The only changes concern the terms $\mathbb{E}\left(I_{1}\right)$ and $\mathbb{E}\left(K_{2}\right)$. Recall that $I_{1}=\left(X_{n}^{2}-\mathbb{E}\left(X_{n}^{2}\right)\right)\left|S_{n-1}\right|^{p-2}$, and let $D_{k}=d_{1}+d_{2}+\cdots+d_{k}$. Since $\mathbb{E}\left(\left(X_{n}^{2}-\right.\right.$ $\left.\left.\mathbb{E}\left(X_{n}^{2}\right)\right)\left|D_{n-1}\right|^{p-2}\right)=0$, we have

$$
\mathbb{E}\left(I_{1}\right)=\mathbb{E}\left(\left(X_{n}^{2}-\mathbb{E}\left(X_{n}^{2}\right)\right)\left(\left|S_{n-1}\right|^{p-2}-\left|D_{n-1}\right|^{p-2}\right)\right) .
$$

Let $Z_{k, j}=D_{j}+\sum_{i=1}^{k}\left(X_{i}-d_{i}\right)$, with the convention $Z_{0, j}=D_{j}$. Then

$$
\mathbb{E}\left(I_{1}\right)=\mathbb{E}\left(\sum_{k=1}^{n-1}\left(X_{n}^{2}-\mathbb{E}\left(X_{n}^{2}\right)\right)\left(\left|Z_{k, n-1}\right|^{p-2}-\left|Z_{k-1, n-1}\right|^{p-2}\right)\right) .
$$

Taking the conditional expectation with respect to $\mathcal{F}_{k}$ and using that $\left.|| x\right|^{p-2}-$ $|y|^{p-2}|\leq| x-\left.y\right|^{p-2}$, we obtain that

$$
\left|\mathbb{E}\left(I_{1}\right)\right| \leq \sum_{k=1}^{n-1}\left\|\mathbb{E}\left(X_{n}^{2}-\mathbb{E}\left(X_{n}^{2}\right) \mid \mathcal{F}_{k}\right)\left|X_{k}-d_{k}\right|^{p-2}\right\|_{1}
$$

This inequality (0.1) must be used instead of the inequality (3.2) of the previous proof. In the same way,

$$
\begin{aligned}
\mathbb{E}\left(K_{2}\right)= & (p-1) \mathbb{E}\left(\sum _ { k = n - N + 1 } ^ { n - 1 } \sum _ { i = 1 } ^ { ( 2 k - n ) _ { + } } ( X _ { n } X _ { k } - \mathbb { E } ( X _ { n } X _ { k } ) ) \left(\left|Z_{i,(2 k-n)_{+}}\right|^{p-2}\right.\right. \\
& \left.\left.-\left|Z_{i-1,(2 k-n)_{+}}\right|^{p-2}\right)\right) .
\end{aligned}
$$

Taking the conditional expectation with respect to $\mathcal{F}_{i}$ and using that $\left.|| x\right|^{p-2}-|y|^{p-2} \mid \leq$ $|x-y|^{p-2}$, we obtain that 


$$
\left|\mathbb{E}\left(K_{2}\right)\right| \leq(p-1) \sum_{k=n-N+1}^{n-1} \sum_{i=1}^{(2 k-n)_{+}}\left\|\mathbb{E}\left(X_{n} X_{k}-\mathbb{E}\left(X_{n} X_{k}\right) \mid \mathcal{F}_{i}\right)\left|X_{i}-d_{i}\right|^{p-2}\right\|_{1}
$$

This inequality (0.2) must be used instead of the inequality (3.5) of the previous proof.

Once we have replaced (3.2) by (0.1) and (3.5) by (0.2), the proof of the new version of Proposition 3.1 is exactly the same as the proof of the old version of Proposition 3.1.

\section{Correction of the proof of Theorem 2.1}

We use the same notations as in the previous proof. Everything is exactly identical up to Inequality (2.14) of the previous proof. After (2.14), we proceed as follows.

We now control the term $\left.\left.\mathbb{E}\left(\mid Z_{n}(](i-1) 2^{-L}, i 2^{-L}\right]\right)\left.\right|^{p}\right)$ with the help of the new Proposition 3.1. Let $T_{i, k}=\mathbf{1}_{(i-1) 2^{-L}<Y_{k} \leq i 2^{-L}}$ and $T_{i, k}^{(0)}=T_{i, k}-\mathbb{E}\left(T_{i, k}\right)$. We apply the new Remark 3.1 to the stationary sequence $\left(T_{i, k}^{(0)}\right)_{k \in \mathbb{Z}}$, by taking $d=-\mathbb{E}\left(T_{i, k}\right)$ (hence $\left|T_{i, 0}^{(0)}-d\right|=\left|T_{i, 0}\right|$ ). We obtain that

$$
\begin{aligned}
\left.\left.\mathbb{E}\left(\mid Z_{n}(](i-1) 2^{-L}, i 2^{-L}\right]\right)\left.\right|^{p}\right) & =\frac{1}{n^{p / 2}} \mathbb{E}\left(\left|\sum_{k=1}^{n} T_{i, k}^{(0)}\right|^{p}\right) \\
& \leq C\left(a_{i}^{p / 2}+n^{(2-p) / 2}\left(\left\|T_{i, 0}^{(0)}\right\|_{p}^{p}+c_{i, 1}+c_{i, 2}+c_{i, 3}\right)\right),
\end{aligned}
$$

where, for any $1 \leq N \leq n$,

$$
\begin{aligned}
a_{i} & =\frac{1}{2} \operatorname{Var}\left(T_{i, 0}\right)+\sum_{k=1}^{N-1}\left|\operatorname{Cov}\left(T_{i, 0}, T_{i, k}\right)\right|+\sum_{k=N}^{n-1}\left\|T_{i, 0}^{(0)} \mathbb{E}\left(T_{i, k}^{(0)} \mid \mathcal{M}_{0}\right)\right\|_{p / 2}, \\
c_{i, 1} & =\sum_{l=1}^{N-1} \sum_{k=0}^{l}\left\|\left|T_{i, 0}^{(0)}\right|^{p-2}\left|T_{i, k}^{(0)}\right| \mathbb{E}\left(T_{i, k+l}^{(0)} \mid \mathcal{M}_{k}\right)\right\|_{1}, \\
c_{i, 2} & =\sum_{l=1}^{N-1} \sum_{k=l}^{n}\left\|\left|T_{i, 0}\right|^{p-2} \mathbb{E}\left(T_{i, k}^{(0)} T_{i, k+l}^{(0)}-\mathbb{E}\left(T_{i, k}^{(0)} T_{i, k+l}^{(0)}\right) \mid \mathcal{M}_{0}\right)\right\|_{1}, \\
c_{i, 3}= & \frac{1}{2} \sum_{k=1}^{n-1}\left\|\left|T_{i, 0}\right|^{p-2} \mathbb{E}\left(\left(T_{i, k}^{(0)}\right)^{2}-\mathbb{E}\left(\left(T_{i, k}^{(0)}\right)^{2}\right) \mid \mathcal{M}_{0}\right)\right\|_{1} .
\end{aligned}
$$

The term $a_{i}$ is the same as in the previous version, and can be handled in the same way. Hence, the inequalities (2.15) and (2.16) of the previous version hold true. After (2.16), we proceed as follows [using the correct definition of $b_{2}\left(\mathcal{M}_{l}, i, j\right)$, as recalled in Paragraph 1 of this erratum, for the control of the term $\left.c_{i, 2}\right]$. 
For the term $c_{i, 1}$, since $\left|T_{i, 0}^{(0)}\right|^{p-2} \leq 1$ and $\sum_{i=1}^{2^{L}}\left|T_{i, k}^{(0)}\right| \leq 2$, one gets

$$
\begin{aligned}
n^{(2-p) / 2} \sum_{i=1}^{2^{L}} c_{i, 1} & \leq 2 n^{(2-p) / 2} \sum_{l=1}^{N} \sum_{k=0}^{l} \sum_{i=1}^{2^{L}} \mathbb{E}\left(\left|T_{i, k}^{(0)}\right| b_{1}\left(\mathcal{M}_{k}, l\right)\right) \\
& \leq 4 n^{(2-p) / 2} \sum_{l=1}^{N}(l+1) \beta_{1}(l) .
\end{aligned}
$$

For the term $c_{i, 2}$, since $\left|T_{i, 0}\right|^{p-2}=T_{i, 0}$ and $\sum_{i=1}^{2^{L}} T_{i, 0}=1$, one gets

$$
\begin{aligned}
n^{(2-p) / 2} \sum_{i=1}^{2^{L}} c_{i, 2} & \leq 4 n^{(2-p) / 2} \sum_{l=1}^{N-1} \sum_{k=1}^{n} \sum_{i=1}^{2^{L}} \mathbb{E}\left(T_{i, 0} b_{2}\left(\mathcal{M}_{0}, k, k+l\right)\right) \\
& \leq 4 n^{(2-p) / 2} N \sum_{k=1}^{n} \beta_{2}(k)
\end{aligned}
$$

For the term $c_{i, 3}$, note first that $\left(T_{i, k}^{(0)}\right)^{2}-\mathbb{E}\left(\left(T_{i, k}^{(0)}\right)^{2}\right)=\left(1-2 \mathbb{E}\left(T_{i, k}\right)\right) T_{i, k}^{(0)}$. Since $\left|1-2 \mathbb{E}\left(T_{i, k}\right)\right| \leq 1$, it follows that

$$
\left|\mathbb{E}\left(\left(T_{i, k}^{(0)}\right)^{2}-\mathbb{E}\left(\left(T_{i, k}^{(0)}\right)^{2}\right) \mid \mathcal{M}_{0}\right)\right| \leq\left|\mathbb{E}\left(T_{i, k}^{(0)} \mid \mathcal{M}_{0}\right)\right| \leq 2 b_{1}\left(\mathcal{M}_{0}, k\right) .
$$

Hence, since $\left|T_{i, 0}\right|^{p-2}=T_{i, 0}$ and $\sum_{i=1}^{2^{L}} T_{i, 0}=1$, one gets

$$
\begin{aligned}
n^{(2-p) / 2} \sum_{i=1}^{2^{L}} c_{i, 3} & \leq 2 n^{(2-p) / 2} \sum_{k=1}^{n} \sum_{i=1}^{2^{L}} \mathbb{E}\left(T_{i, 0} b_{1}\left(\mathcal{M}_{0}, k\right)\right) \\
& \leq 2 n^{(2-p) / 2} \sum_{k=1}^{n} \beta_{1}(k)
\end{aligned}
$$

Note that the last bounds on the right hand side of $(0.3),(0.4)$ and $(0.5)$ are exactly the same as the upper bounds (2.17), (2.18) and (2.19) of the previous version, and so the proof of Theorem 2.1 can be completed as previously.

Acknowledgments I wish to thank Florence Merlevède who pointed out both the problem in the definition of $\beta_{2}(n)$, and the wrong argument in the proof of Theorem 2.1.

\section{Reference}

1. Dedecker, J., Prieur, C.: An empirical central limit theorem for dependent sequences. Stoch. Process. Appl. 117, 121-142 (2007) 